\title{
Generic Nanomaterial Positioning by Carrier and Stationary Phase Design
}

2007

Vol. 7, No. 9

2764-2768

\author{
Roie Yerushalmi, Johnny C. Ho, Zachery A. Jacobson, and Ali Javey* \\ Department of Electrical Engineering and Computer Sciences, University of California \\ at Berkeley, Berkeley, California 94720, and Materials Science Division, \\ Lawrence Berkeley National Laboratory, Berkeley, California 94720
}

Received May 29, 2007; Revised Manuscript Received July 19, 2007

\begin{abstract}
A generic approach for deploying various types of synthetic nanomaterials, including single-walled carbon nanotubes and nanoparticles, at well-defined locations on substrates is presented. The assembly is achieved through the complementary design of the stationary and carrier phases and utilizes the dewetting process during which polymer-encapsulated nanomaterials are delivered and positioned on predefined locations of substrates. Covalent modification of the nano-objects is not required for the building block positioning, therefore, preserving their intrinsic chemical and physical properties. The potency of this new approach is demonstrated for various synthetic nanomaterials, such as polystyrene, silica, and gold nanoparticles as well as single-walled carbon nanotubes, showing highly specific and direct patterning of $\mathrm{cm}^{2}$ areas using a generic assembly strategy.
\end{abstract}

Nanoscale materials have been the subject of major scientific and technological breakthrough in the past decade. The remarkable advancements in synthetic methods of nanomaterials have resulted in vast diversity of readily accessible materials with highly controlled composition and dimensions. Current approaches for patterning surfaces with nanosized objects include geometrical confinement, well formation techniques, utilization of specific covalent and noncovalent chemical surface interactions, capillary interactions, soft lithography, electrostatic assembly, and more. ${ }^{1-13}$ However, more progress is still needed for uniform, large-scale, and selective deployment of nano-objects of different materials and aspect ratios. Here, we present a highly generic approach for directly deploying various types of synthetic nanomaterial building blocks at well-defined locations on substrates by surface energy engineering. Rather than focusing on the nanomaterial and substrate interactions which are highly material dependent, we have exploited a polymer-assisted route for generic delivery and positioning of various nanomaterials on substrates.

Our approach aims at the complementary design of the stationary phase (chemically patterned substrate) and the carrier phase (block copolymer aqueous-alcohol solution containing the nanomaterials) that guide the assembly of the nanomaterials during the dewetting process (Figure 1). We demonstrate the selective and direct positioning of various nanoparticles, including polystyrene, silica, and gold, as well as single-walled carbon nanotubes (SWNTs) using a similar condition. The nanomaterial is selectively distributed and

\footnotetext{
* Corresponding author. ajavey@eecs.berkeley.edu.
}

positioned on the stationary phase by spontaneous dewetting of the carrier phase. Nanomaterial attachment is polymerassisted by noncovalent interactions at the chemically modified regions of the substrates. Our method does not require covalent chemical surface modification of the nanomaterials, therefore, preserving their intrinsic physical and chemical properties. Furthermore, the assembled materials are not exposed to the resist lift-off process which may degrade or contaminate certain molecular-scale structures. The potency of this approach is demonstrated as equal treatment of various synthetic nanomaterials results in highly specific and direct patterning of $\mathrm{cm}^{2}$ areas.

The stationary phase consisted of a silicon (100) substrate with $50 \mathrm{~nm}$ thermal oxide layer that was chemically patterned by well-established siloxane-based condensation chemistry ${ }^{14,15}$ and standard lithographic techniques to define hydroxyl-terminated (bis(2-hydroxyethyl)-(3-aminopropyl)triethoxysilane, HEA) and fluoro-terminated ((heptadecafluoro1,1,2,2-tetrahydrodecyl)dimethylchlorosilane, HDF) regions (Figure 1a and Supporting Information). As-received gold, silica, and polystyrene nanoparticles and SWNTs (arcdischarge) were used to prepare aqueous - methanol $(1: 1 \mathrm{v} / \mathrm{v})$ solutions containing the water-soluble triblock copolymer (Pluronic F-127) as the dispersing mediator. To achieve deployment of the nano-objects, the carrier phase containing the respective nanomaterials was retracted swiftly with a pipet (Figure 1b) from a vial containing the chemically patterned substrate. Once the liquid level declined below the substrate surface, spontaneous and rapid dewetting of the solution phase from the highly hydrophobic HDF modified 
(a)

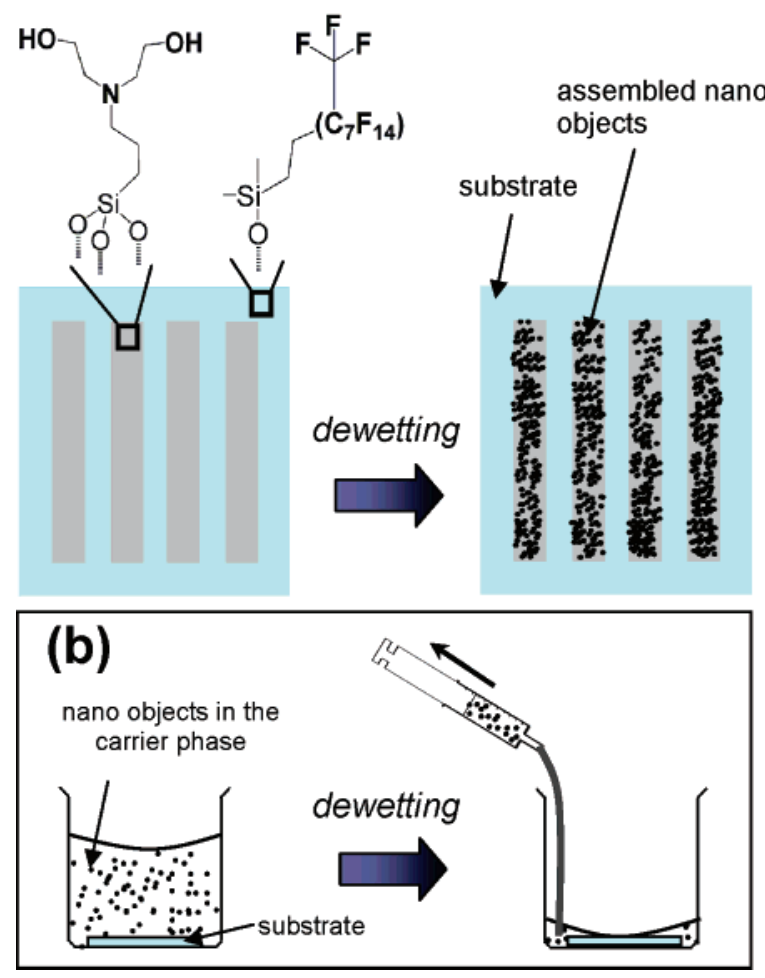

Figure 1. Illustration of the synthetic nanomaterial positioning procedure and surface functionalization. (a) $\mathrm{A} \mathrm{Si} / \mathrm{SiO}_{2}$ substrate is chemically modified with fluoro-terminated (light blue) and hydroxyl-terminated (gray) regions that serve as the stationary phase. After the dewetting process, the nano-objects from the carrier phase are directly and selectively assembled on hydroxyl-terminated regions while leaving the fluoro-terminated regions blank. (b) To achieve the dewetting process, the carrier phase containing the nanomaterials and the triblock F-127 polymer is swiftly retracted by a pipet from a vial until the liquid level drops below the surface of the substrate. At this point, rapid and spontaneous deployment of the nano-objects to the predefined hydroxyl-terminated regions is achieved.

regions occurred. Notably, the dewetting occurred for the entire chip in a subsecond time scale, with the vial still retaining the vapor-liquid equilibrium. Thus, simple liquid evaporation can be excluded as the driving force for the nanomaterial deposition. ${ }^{16,17}$ The gold $(30 \mathrm{~nm})$, silica $(50$ $\mathrm{nm})$, and polystyrene $(50 \mathrm{~nm})$ particles show highly selective positioning at the HEA-modified areas with almost no particles assembled at the HDF modified regions (Figure 2). Uniform results are achieved over large scales as evident from the dark field optical microscope image (Figure 2a). The dewetting induced forces direct the polymer-encapsulated particles to the patterned hydroxyl-functionalized areas with various shapes and dimensions ranging from $\sim 200 \mathrm{~nm}$ to $10 \mu \mathrm{m}$ (Figure $2 \mathrm{~b}-\mathrm{f}$ ). Notably, the nanoparticles were assembled without any significant multilayer aggregation (Figure 2c,d,f). These results are distinct from the nanoparticle assembly attained by liquid evaporation mechanisms where nanoparticle-nanoparticle and capillary interactions lead to multilayer aggregation and ordered pattern formation. $3,11 \mathrm{~b}, 16,17$
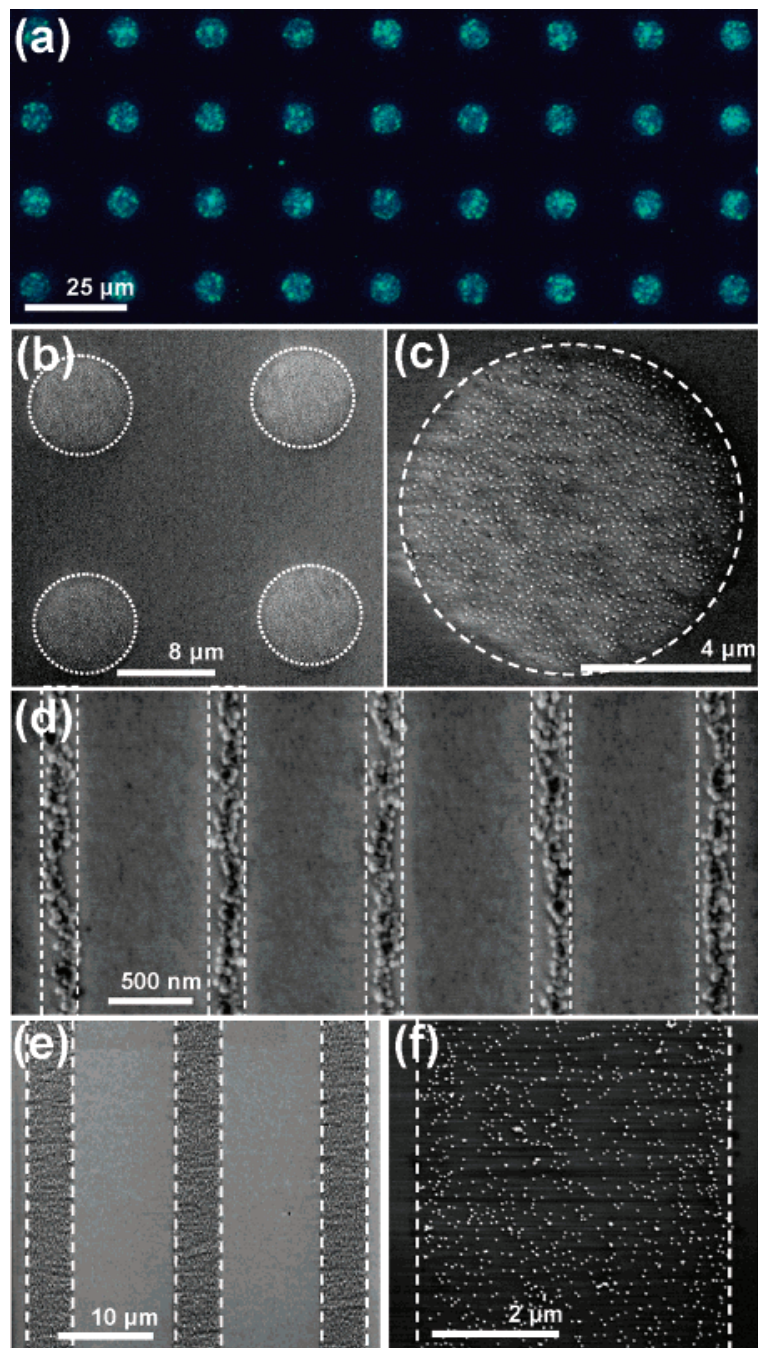

Figure 2. Patterned nanoparticle assembly using 0.5\% Pluronic F-127 polymer solution. (a) Dark field optical image and (b-d) scanning electron microscopy images of $50 \mathrm{~nm}$ polystyrene nanoparticles positioned in linear and circular geometries. (e, f) SEM image of the assembled $30 \mathrm{~nm}$ gold nanoparticles. Dashed lines indicate the HEA-modified areas.

To exhibit the role of polymer interactions in the dewetting process, we performed quantitative nanoparticle assembly experiments with unmodified silica nanoparticles for various particle and polymer concentrations (Figure 3). When the dewetting process is conducted without the use of F-127 polymer, almost no nanoparticle (NP) assembly $(<0.01 \mathrm{NP} /$ $\left.\mu \mathrm{m}^{2}\right)$ is observed even for very high nanoparticle solution concentrations (Figure 3a,c). This is in distinct difference with the dewetting process that is conducted under the exact same conditions but with the addition of F-127 polymer to the carrier phase. In this case, a dense monolayer of nanoparticles $\left(\sim 100 \mathrm{NP} / \mu \mathrm{m}^{2}\right)$ is selectively assembled at the hydroxyl-terminated regions (Figure 3b). This clearly demonstrates that the direct chemical interactions between the unmodified silica nanoparticles and the hydroxyl-terminated surfaces are very weak and not adequate for collecting the particles from the solution phase under these conditions. Therefore, the assembly can only be achieved when Pluronic F-127, which is an amphiphilic block copolymer with well- 


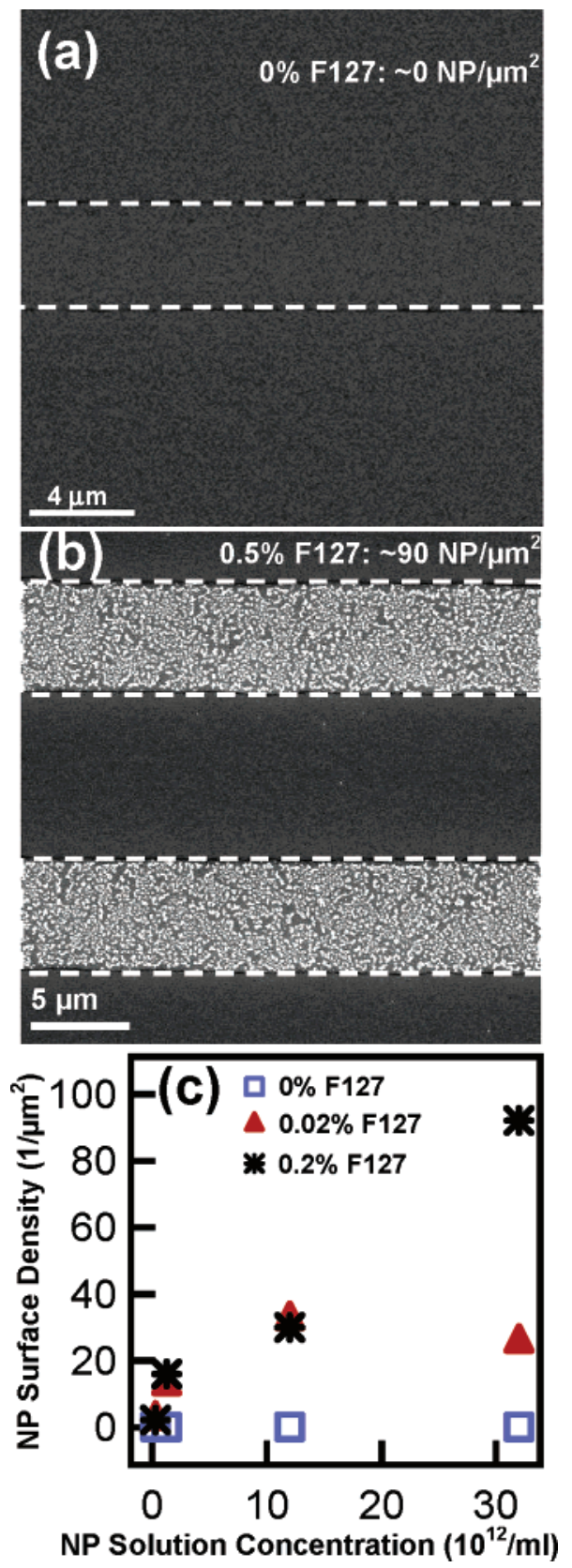

Figure 3. Silica nanoparticle density as a function of polymer concentration. Scanning electron microscopy image of $50 \mathrm{~nm}$ silica nanoparticles positioned in linear geometries using (a) $0 \%$ and (b) $0.5 \%$ Pluronic F-127 polymer solutions, respectively. Dashed lines indicate the HEA-modified areas. (c) Assembeled nanopaticle surface density versus nanoparticle concentration for $0,0.02$, and $0.2 \%$ F-127 polymer solutions.

known colloidal dispersing properties, is used to encapsulate the nanoparticles and serve as a mediator for delivering and positioning the particles. The F-127 polymer consists of poly(ethylene oxide)-poly(propylene oxide) - poly(ethylene oxide) (PEO-PPO-PEO) units. Due to the effective chemical interactions between the PEO units of F-127 and the hydroxyl groups of the substrate, the polymer-encapsulated nanomaterials are anchored to the HEA-modified regions during the dewetting process, therefore, resulting in selective and direct assembly of the particles. Because the assembly is achieved through the complementary design of the chemical interactions between the functionalized substrate and the polymer mediator (rather than the nanomaterials itself), our simple approach is highly generic and versatile for positioning various nanomaterials on substrates, regardless of their composition. $^{22}$

The quantitative trend for the nanoparticle solution concentration versus surface density for three different polymer concentrations is shown in Figure 3c which further shows the key role of the F-127 polymer in guided assembly of the silica particles. While almost no nanoparticle is assembled without the polymer in the carrier phase, at relatively low polymer concentrations $(0.02 \%)$ the assembled nanoparticle surface density approaches saturation level of $\sim 40 \mathrm{NP} / \mu \mathrm{m}^{2}$ (submonolayer) at a solution concentration of $\sim 10^{13} \mathrm{NP} /$ $\mathrm{mL}$, and further increase in nanoparticle solution concentration does not yield a higher surface density. Increasing the polymer concentration from 0.02 to $0.20 \%$ results in monotonic increase in the nanoparticle surface density with the nanoparticle solution concentration for the entire range of particle concentrations that were studied, enabling a density of $\sim 90 \mathrm{NP} / \mu \mathrm{m}^{2}$ for $\sim 3 \times 10^{13} \mathrm{NP} / \mathrm{mL}$. Notably, at low nanoparticle solution concentrations, no significant difference is observed for the two polymer concentrations. This trend shows that while for the low nanoparticle solution concentrations $\left(<10^{13} \mathrm{NP} / \mathrm{mL}\right)$, the system is nanoparticle limited for both $0.2 \%$ and $0.02 \%$ polymer concentrations, at high nanoparticle concentrations $\left(>10^{13} \mathrm{NP} / \mathrm{mL}\right)$, the assembly is polymer-limited for the $0.02 \% \mathrm{~F}-127$ solution. Therefore, the assembly density is well controlled, and the nanoparticle surface density can be tuned by nanoparticle solution concentration as well as the polymer concentration. It is possible to tune the assembly process for attaining the desired surface density by employing a surface density calibration curve, as depicted in Figure 3c, for any specific nanomaterial. Such fine controllability is useful for various applications where different surface densities are highly desired.

Beside the triblock polymer, the solvent plays a critical role in the outcome of the dewetting process. The wateralcohol solution ratio was found to drastically influence the assembly yield of the nanomaterials. For instance, increasing the water content from the optimal 1:1 aqueous-methanol mixture resulted in less effective positioning of the gold nanoparticles with lower density and selectivity. This may be attributed to the enhancement of the H-bond capacity of the solvent as the water content is increased, resulting in stronger solvent interactions with both the polar PEO polymer groups and the $\mathrm{OH}$ functional groups at the HEAmodified surfaces. As a result, the effective polymersubstrate interactions are overwhelmed by the solvent interactions, therefore, hampering the direct and selective positioning of the nanomaterials.

To further demonstrate the generality of the approach, we utilized stable aqueous solutions of SWNTs. The steric stabilization mechanism of Pluronic polymers have been previously reported for SWNTs. ${ }^{18-21}$ Figure 4 presents the SWNT deployment results having similar characteristics to the nanoparticle assembly using a nearly identical process. 


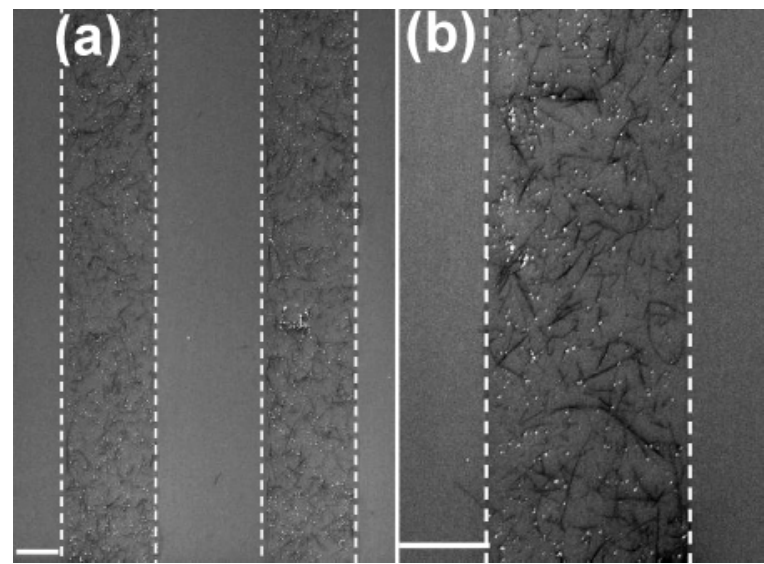

Figure 4. Direct patterning of high aspect ratio nanomaterials using $0.5 \%$ F-127 polymer solution. (a, b) Scanning electron microscopy images of SWNTs selectively positioned in linear geometries. Scale bars are $2 \mu \mathrm{m}$.

This result further demonstrates the generality of our approach of aiming at the carrier phase to stationary phase pairing rather than aiming at the nanomaterials-surface interactions directly. Notably, many approaches that are useful for selective nanoparticle positioning and assembly are not well-suited for nano-objects with high aspect ratio such as nanowires and nanotubes. This is in part because interactions scale differently for nanoscale spherical materials and wirelike structures with nanoscale diameters and microscale lengths. ${ }^{23,24}$ It is important to note that the triblock copolymer aqueous - methanol solution containing the nanoobjects constitutes a complex system with versatile thermodynamic characteristics. Pluronic polymers contain covalently bonded and mutually incompatible moieties. As a result, their aqueous solutions are known to have a wide range of thermodynamically and dynamically accessible states involving micelles, vesicles, and gel phases. ${ }^{25,26}$ Interestingly, although the polymer solution stabilization mechanism details may vary for the different nano-object solutions studied here, the highly selective positioning of the assembled nano-objects without significant aggregation is retained for all materials using the same generic process. The detailed study of such phases and their exact role in the context of the direct nanomaterial selective positioning is currently in progress. Understanding the dynamic nature of these processes is expected to further generalize the application of this complex and yet versatile system.

While the selective nanotube assembly attests to the versatility and generic nature of our polymer-guided assembly strategy, the lack of order in the positioning of the nanotubes presents a disadvantage over certain previously explored routes, such as the nanoaffinity template method. ${ }^{\text {la }}$ However, in the future, single nanotube or nanoparticle positioning may be possible to achieve by scaling down the dimensions of the HEA-modified regions of the substrate which can further enhance the control over orientation and alignment. A unique feature of our approach as compared to most other assembly strategies ${ }^{1-13}$ is its highly generic nature, making it compatible with a wide range of nanostructured materials, regardless of their geometry and com- position. This is achieved as we have exploited a novel polymer-assisted route for encapsulation and deployment of the nanomaterials without focusing on the commonly incorporated nanomaterials-substrate interactions which are highly material dependent. Another key feature of our work is the ability to readily achieve direct nanomaterial assembly with tunable densities, as shown for $50 \mathrm{~nm}$ nanoparticles, with the maximum density approaching $\sim 100 \mathrm{NP} / \mu \mathrm{m}^{2}$ $(\sim 25 \%$ surface coverage), without any significant aggregation arising from the polymer stabilization of the nanomaterials. Furthermore, our strategy may present a novel avenue for direct assembly of organic and biological nanomaterials that are not compatible with processes involving postassembly lift-off process, such as the geometric confinement method. ${ }^{3}$

In summary, we have presented a novel and generic strategy that enables selective positioning of various types of synthetic nanomaterials of different geometry and composition, such as nanoparticles and SWNTs, at predefined locations on the substrate, without aggregation and at large scales. The assembly is achieved through the chemical design of the stationary and carrier phases, and utilizes the dewetting interactions. Importantly, covalent modification of the nanoobjects is not required for the process.

Acknowledgment. We acknowledge financial support from MARCO MSD Focus Center Research Program, and a Human Frontiers Science Program fellowship (R.Y.). All fabrication was performed in the Berkeley Microlab facility.

Supporting Information Available: Procedures for surface modification and nanoparticle and SWNT solution preparation. This material is available free of charge via the Internet at http://pubs.acs.org.

\section{References}

(1) (a) Wang, Y.; Maspouch, D.; Zou, S.; Schatz, G. C.; Smalley, R. E.; Mirkin, C. A. Proc. Natl. Acad. Sci. U.S.A. 2006, 103, 2026-2031. (b) Rao, S. G.; Huang, L.; Setyawan, W.; Hong, S. H. Nature 2003, 425, 36-37. (c) Huang, Y.; Duan, X.; Wei, Q.; Lieber, C. M. Science 2001, 291, 630-633. (d) Whang, D.; Jin, S.; Wu, Y.; Lieber, C. M. Nano Lett. 2003, 3, 1255-1259. (e) Jin, S.; Whang, D. M.; McAlpine, M. C.; Friedman, R. S.; Wu, Y.; Lieber, C. M. Nano Lett. 2004, 4, 915-919.

(2) (a) Javey, A.; Dai, H. J. J. Am. Chem. Soc. 2005, 127, 11942-11943 (b) Wang, D. W.; Chang, Y. L.; Liu, Z.; Dai, H. J. J. Am. Chem. Soc. 2005, 127, 11871-11875. (c) Javey, A.; Nam, S.; Friedman, R. S.; Yan, H.; Lieber, C. M. Nano Lett. 2007, 7, 773-777.

(3) Cui, Y.; Bjork, M. T.; Liddle, J. A.; Sonnichsen, C.; Boussert, B.; Alivisatos, P. Nano Lett. 2004, 4, 1093-1098.

(4) Barsotti, R. J.; Stellaci, F. J. Mater. Chem. 2006, 16, 962-965.

(5) Yin, Y.; Lu, Y.; Xia, Y. J. J. Am. Chem. Soc. 2001, 123, 771-772.

(6) Wooley, K. L.; Hawker, C. J. Top. Curr. Chem. 2005, 245, 287305.

(7) Maenosono, S.; Okubo, T.; Yamaguchi, Y. J. Nanopart. Res. 2003, $5,5-15$.

(8) Michel, R.; Reviakine, I.; Sutherland, D.; Foaks, C.; Csus, G.; Danuser, G.; Spencer, N. D.; Textor, M. Langmuir 2002, 18, 85808586.

(9) Hannon, J. B.; Afzali, A.; Klinke, C.; Avouris, P. Langmuir 2005, $21,8569-8571$.

(10) Li, S. P.; Russell, D. M.; Newsome, C. J.; Kugler, T. Appl. Phys. Lett. 2006, 89, 122105.

(11) (a) Huang, J.; Tao, A. R.; Connor, S.; He, R.; Yang, P. Nano Lett. 2006, 6, 524-529. (b) Huang, J.; Kim, F.; Tao, R. T.; Connor, S.; Yang, P. Nat. Mater. 2005, 4, 896-900. 
(12) (a) Sun, Y.; Rogers, J. A. Nano Lett. 2004, 4, 1953-1959. (b) Meitl, M. A.; Zhou, Y. X.; Gaur, A.; Jeon, S.; Usrey, M. L.; Strano, M. S.; Rogers, J. A. Nano Lett. 2004, 4, 1643-1647.

(13) Barry, C. R.; Gu, J.; Jacobs, H. O. Nano Lett. 2005, 5, 20782084.

(14) Gulino, A.; Condorelli, G. G.; Mineo, P.; Fragala, I. Nanotechnology 2005, 16, 2170-2175.

(15) Yerushalmi, R.; Scherz, A.; van der Boom, M. E. J. Am. Chem. Soc. 2004, 126, 2700-2701.

(16) Celio, H.; Barton, E.; Stevenson, K. J. Langmuir 2006, 22, 1142611435 .

(17) Choi, W. M.; Park, O. O. Colloids Surf., A 2006, 277, 131-135.

(18) Sakai, T.; Alexandridis, P. Langmuir 2004, 20, 8426-8430.

(19) Shvartzman-Cohen, R.; Levi-Kalisman, Y.; Nativ-Roth, E.; YerushalmiRozen, R. Langmuir, 2004, 20, 6085-6088.

(20) Moore, V. C.; Strano, M. S.; Haroz, E. H.; Schmidt, J.; Talmon, Y.; Hauge, R. H.; Smalley, R. E. Nano Lett. 2003, 3, 1379.

(21) (a) Lin, Y.; Alexandridis, P. J. Phys. Chem. B 2002, 106, 1083410844. (b) Lin, Y.; Smith, T. W.; Alexandridis, P. J. Dispersion Sci. Technol. 2002, 23, 539-553. (c) Kim, J.; Koffas, T. S.; Lawrence,
C.C.; Somorjai, G. A. Langmuir 2004, 20, 4640-4646. (d) Iqbal, M.; Chung, Y.; Tae, G. J. Mater. Chem. 2007, 17, 335-342. (e) Nativ-Roth, E.; Shvartzman-Cohen, R.; Bounioux, C.; Florent, M.; Zhang, D.; Szleifer, I.; Yerushalmi-Rozen, R. Macromolecules 2007, 40, 3676-3685.

(22) We speculate that after the dewetting process, a monolayer of physisorbed triblock polymer is left on the surface of the nanomaterials which is not observable with scanning electron microscopy. For the purpose of this work, no postassembly treatment was conducted to remove the polymer residues. In the future, if desired, a solvent- or gas-based process may be applied to selectively remove the polymer.

(23) Szleifer, I.; Yerushalmi-Rozen, R. Polymer 2005, 46, 7803-7818.

(24) KralchevskyI, P. A.; Nagayama, K. Adv. Colloid Interface Sci. 2005, $85,145-192$.

(25) Soo, P. L.; Eisenberg, A. J. Polym. Sci. 2004, 42, 923-938.

(26) Kwon, K. W.; Park, M. J.; Hwang, J.; Char, K. Polym. J. 2001, 33, 404-410.

NL071271B 


\title{
Generic Nano-material Positioning by Carrier and Stationary Phase Design
}

\author{
Roie Yerushalmi, Johnny C. Ho, Zachery A. Jacobson, and Ali Javey* \\ Department of Electrical Engineering and Computer Sciences, University of \\ California at Berkeley, Berkeley, CA 94720. Materials Science Division, \\ Lawrence Berkeley National Laboratory, Berkeley, CA 94720
}

Supporting Information 


\section{Experimental}

Silicon (100) substrates with 50nm thermally grown oxide layer were reacted with (Heptadecafluoro-1,1,2,2-tetrahydrodecyl) dimethylchlorosilane, 0.5 vol\% in hexane at room temperature for 45 min to form a fluoro terminated surface. The substrates were then thoroughly washed with copious amounts of dry hexane and dried at $120^{\circ} \mathrm{C}$ for 20 min. Subsequently, photolithography (Shipley 1805 resist) was used to form micron size patterns on the chemically reacted substrates. For sub-micron features electron beam lithography was employed using a Poly(methyl methacrylate) (PMMA, Micro Chem) resist. After development of the resist, the exposed areas were etched by a mild oxygen plasma ( 0.28 torr, $40 \mathrm{~W}$ power, $50 \mathrm{sccm} \mathrm{O}_{2}$ flow rate for $60 \mathrm{sec}$ ) to remove the fluoroterminated surface modification followed by resist removal in acetone, and drying at $120^{\circ} \mathrm{C}$ for $20 \mathrm{~min}$. Then, the samples were reacted with bis(2-hydroxyethyl)-3aminopropyltriethoxysilane, $0.5 \mathrm{vol} \%$ in ethanol, resulting in the formation of the hydroxyl terminated surfaces on the areas that were previously exposed to $\mathrm{O}_{2}$ plasma. The formation of monolayers was confirmed by advancing contact angle and ellipsometry measurements.

Water-soluble polymer [poly(ethylene oxide)106-poly(propylene oxide)70poly(ethylene oxide)106, PEO-PPO-PEO (Pluronic ${ }^{\circledR}$ F-127), of molecular weight 12,500 g/mol was dissolved in DI:MeOH $(1: 1 \mathrm{v} / \mathrm{v})$ to form $0.02-2.00 \%$ polymer solutions and was refrigerated for two days prior to use. Distilled and deionized water (DI) was used for the preparation of all solutions.

Nanoparticle solution of polystyrene (50 nm from Polysciences, Inc.), silica (50 nm from Polysciences, Inc.), and gold colloid (unconjugated, 30 nm from Ted Pella, Inc.) 
were prepared by adding the polymer solution to methanol, followed by the addition of equivalent amount of aqueous colloid solution, resulting in $0.5 \mathrm{wt} \%$ polymer in DI:MeOH (1:1 v/v).

To prepare the SWNT solution, $20 \mathrm{mg}$ of as-received arc-discharged SWNTs (Sigma CarboLex AP-grade) was sonicated in $8 \mathrm{ml}$ of the polymer solution for $10 \mathrm{~min}$ using a bath sonicator. The resulting dispersion was then centrifuged at $14,000 \mathrm{~g}$ for 10 min, and the supernatant collected and sonicated for 3 min. 